\title{
Percutaneous or Open Release is the Most Effective Surgical Technique in Diabetic Recurrent Trigger Finger in Short and Long Term Outcomes? A Clinical Review
}

\section{Grigorios Kastanis ${ }^{1 *}$, Pantouvaki $\mathrm{A}^{2}$, Spyrantis $\mathbf{M}^{1}$, Christoforidis $\mathrm{C}^{\mathbf{1}}$ and Velivasakis $\mathbf{G}^{\mathbf{1}}$}

${ }^{1}$ Department of Orthopaedic, General Hospital of Heraklion-Venizeleio, Crete, Greece

${ }^{2}$ Department of Physiotherapy, General Hospital of Heraklion-Venizeleio, Crete, Greece

*Corresponding Author: Grigorios Kastanis, Department of Orthopaedic, General Hospital of Heraklion-Venizeleio, Crete, Greece.
Received: March 27, 2020

Published: April 21, 2020

(c) All rights are reserved by Grigorios

Kastanis., et al.

\begin{abstract}
Introduction: Stenosing Tenosynovitis is a common disorder among patients with diabetes mellitus. The definitive treatment is release of the A1 pulley (open or percutaneous). The aim of this study is to compare functional outcomes and complication rates of these two methods (open versus percutaneous release) for recurrent trigger finger in patients with diabetes mellitus.

Materials and Methods: 114 patients with diabetes mellitus and an average age of 48 years (range 28 - 64 yrs old) were treated for recurrent trigger finger. The modified Quinnell grading system was used as a selection criterion for the classification of trigger digit. The patients were divided in two groups randomly. Group A included 52 patients who were treated with open release of A1 pulley and group B included 62 patients who were treated with percutaneous release using the tip of an 18-gauge or knife (Ophthalmic Corneal/Scleral knife 19 Gauge).

Results: The median follow-up was 12 months (range from 10 - 14 months). The results were based on the Visual Analogue Scale (VAS), Quick Dash and Gilberts and Wereldasma Questionnaire. At 3 months, the results seemed to be better in group B, while there was no statistical difference at six months and at one year postoperatively.

Conclusion: Both surgical methods (open and percutaneous release) in long-term follow-up postoperatively, resulted in similar therapeutic efficacy. The advantages of percutaneous release over the classic open method in short-term outcomes are: 1) low cost,

2) less complication rates and 3) immediate functional recovery.

Keywords: Percutaneous Release; Open Release; Diabetes Mellitus; Trigger Finger
\end{abstract}

\section{Introduction}

Stenosing Tenosynovitis or trigger finger (TF) is caused by the discrepancy between the volume of the flexor tendon sheath and its contents, resulting in a narrowed tunnel for the flexors excursion. This leads to a symptomatic block of the digit during movement. Triggering most commonly involves the A1 pulley owing to high pressure gradient and high local forces in this area at maximal flexion and tight hand grip [1]. The incidence of trigger finger is about 28 cases per 100.000 population per year, with a lifetime risk of $2.6 \%$ in the general population, while in patients with diabetes mellitus (DM) is $10 \%[2,3]$.

The plurality of trigger finger is idiopathic, while a small proportion belongs into secondary group with diabetes mellitus, kidney disease, amyloidosis, rheumatoid arthritis, mucopolysaccharidosis and repeated minor trauma to the hand [4]. The average age of appearance of TF is 50 - 60 years old, with females having a risk of 2 - 6 times more than men [5]. The thumb is the most common involved digit followed by the ring finger, long, small and finally the index finger. In female the most common affected digit is thumb
[6]. The prevalence of TF in patients with DM varied from 5 - 10\% and is common in old diabetic patients but not related to sex, age and type of diabetes. The main characteristic of TF in the group of patients with DM, is the involvement of multiple digits in one third of them [7].

Multiple conservative treatment methods (activity modification, passive stretching of fingers, non-steroidal anti-inflammatory drugs, hydrotherapy, splinting of the finger, corticosteroid injection) provide complete relief of symptoms especially in early stage. When conservative therapy failures, the surgical (open or subcutaneous) release of A1 pulley is mandatory, with the aim of full functional recovery of the digit. Open release of A1 pulley has a success rate of $99 \%$, while in percutaneous release (P.R.) the rate ranges from $74 \%$ to $94 \%[8,9]$.

\section{Aim of the Study}

The aim of this study is to compare functional outcomes and complication rates of conventional open release versus percutaneous, for recurrent trigger finger in patients with diabetes mellitus in short (3 months) and long-term (12 months) period. 


\section{Materials and Methods}

The study was performed at the Orthopaedic department of General Hospital of Heraklion-"Venizeleio" from January 2017 to April 2018. The Institutional Ethical Committee approved the study. Inclusion criteria were: adults more than 20 years old with controlled diabetes mellitus and Quinnell grade I-IV (Table 1), who failed in conservative treatment, with a duration of symptoms more than 3 months. Exclusion criteria were: Uncontrolled diabetes mellitus, immunosuppressive diseases, modified Quinnell grade I, rheumatoid arthritis, previous hand trauma or surgery, a neurologic deficit in the same upper extremity. One hundred fourteen patients were included in this study. Seventy three were females and forty one males, with an average of 48 years old (range 28 - 64 yrs old). The mean duration of symptoms before release was 11.4 months (range 5 -16 months). Diabetes was defined as type I (in 34 cases) or type II (in 80 cases) according to the American Diabetes Association (ADA) criteria. All patients preoperatively were examined clinically to classify the grade of TF (Modified Quinnell grading system), blood test examination (blood sugar) and x-rays of the affected hand. Multiple digits involved in 45 patients (27 cases involved thumb and middle finger, while 18 cases involved ring and middle finger) and 69 cases presented with one digit. Patients were randomly divided in two groups. There was no statistical difference in homogeneity of the group (sex, gender, hand dominance) (Table 2). Group A consisted of 52 patients $(45,7 \%)$ who were treated with open release of A1 pulley and Group B consisted of 62 patients $(54,3 \%)$ who were treated with percutaneous release (19 gauge needle (26 cases) and V - Lance knife 19 Gauge (32 cases)). Both procedures were done in the outpatient department under tourniquet and local anesthesia using 2- $4 \mathrm{cc}$ of $2 \%$ xylocaine. After painting and draping of the affected hand, the affected finger was hyperextended, with the scope the flexor tendon sheath to be directly under the skin.

\begin{tabular}{|c|c|c|}
\hline Grade & Group A (52) & Group B (62) \\
\hline $\begin{array}{c}\text { I: Pain and tenderness over } \\
\text { the A1 pulley with no } \\
\text { entrapment or catching }\end{array}$ & $7(13,4 \%)$ & $8(12,9 \%)$ \\
\hline $\begin{array}{c}\text { II: Visible entrapment but the } \\
\text { patient is able to extend the } \\
\text { finger actively }\end{array}$ & $13(25 \%)$ & $10(16,1 \%)$ \\
\hline $\begin{array}{c}\text { III (A): Entrapment needs } \\
\text { passive extension }\end{array}$ & $12(23,2 \%)$ & $16(25,8 \%)$ \\
\hline $\begin{array}{c}\text { III (B): Inability to actively } \\
\text { flex the finger }\end{array}$ & $15(28,8 \%)$ & $19(30,7 \%)$ \\
\hline IV: Fixed flexion contracture \\
of the finger
\end{tabular}

Table 1: Modified Quinnell grading system.

In open release procedure the landmarks of A1 pulley were delimited according to the Wilhelmi., et al [10]. Skin incision was done over A1 pulley, the neurovascular elements were protected and a longitudinal incision of pulley was achieved. Patients perform flexion-extension of the digit. The skin was closed with 4/0 nylon sutures. Finally, a soft bulky dress was applied leaving the interphalangeal joints free for early motion. The sutures were removed at two weeks.

\begin{tabular}{|l|c|c|c|}
\hline \multicolumn{1}{|c|}{$\begin{array}{c}\text { Patient } \\
\text { No }\end{array}$} & $\begin{array}{c}\text { Group A } \\
\mathbf{5 2}\end{array}$ & $\begin{array}{c}\text { Group B } \\
\mathbf{6 2}\end{array}$ & P \\
\hline Mean age (years) & $40(28-60)$ & $42(30-64)$ & 0,102 \\
Gender & $18 / 34$ & $23 / 39$ & 0,6 \\
(Male/Female) & $(34,7 \% / 65,3 \%)$ & $(37,1 \% / 62,9 \%)$ & \\
Diabetes Mellitus & & & 0,678 \\
Type I & 14 & 20 & \\
Type II & 38 & 42 & \\
Hand involved & $34 / 18$ & $37 / 19$ & 0,898 \\
Right/Left & $(65,4 \% / 34,6 \%)$ & $(59,7 \% / 40,3 \%)$ & \\
Hand dominance & & & 0,320 \\
Right/Left & $42 / 10$ & $44 / 18$ & \\
Single Digit & 24 & 45 & \\
Multiple Digit & 20 & 23 & \\
Digit involved & & 12 & \\
Thumb & 5 & 23 & \\
Index & 9 & 19 & \\
Middle & 21 & & \\
Ring & 17 & & \\
\hline
\end{tabular}

Table 2: Demographic characteristics of both treated group.

Group A. Open release Group B. Percutaneous release.

In percutaneous release group the landmarks were applied according to Hazani., et al. technique, then a needle gauge 19 or V-Lance knife 19 Gauge were introduced over A1 pulley [11]. The A1 pulley is sectioned from distal to proximal. Relief of resistance on the knife tip secure, release the pulley. The unrestrained range of motion of affected digit and disappearance of trigger lesion proves the release of the pulley (Figure 1a-1d). In thumb, the knife or gauge inserted at $1 \mathrm{~cm}$ distally to metacarpophalangeal crease, and the section of the pulley is not overlied proximally to avoid the damage of the radial digital nerve. In all patients of this group after the therapeutic procedure, corticosteroid injections were administrated. (Betamethasone Acetate and Betamethasone) A soft bulky dress was also applied after the end of the procedure.

In both groups postoperatively, patients were advised to use ice packs 3 times per day, for three to four days, with focus to avoid postoperative edema.

Postoperatively, all patients underwent an exercise program of passive and active movements as well as stretches to reduce edema, stiffness and to enhance functional rehabilitation of the hand. Patients were attended for physiotherapy treatment twice a week and were trained to perform their exercises four times daily.

\section{Statistical analysis}

Patients were assessed for all variables. The chi-square test was used to compare differences between two group of treatment $(\mathrm{p}$ 
(a)

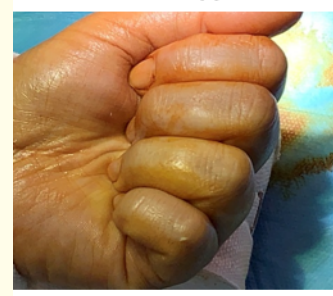

(c)

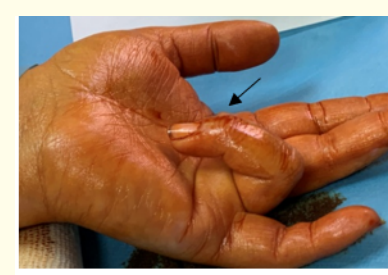

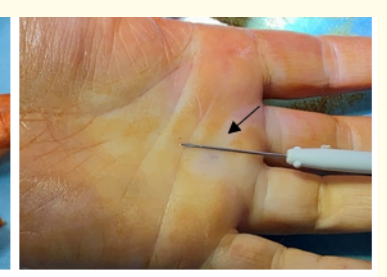

(b)

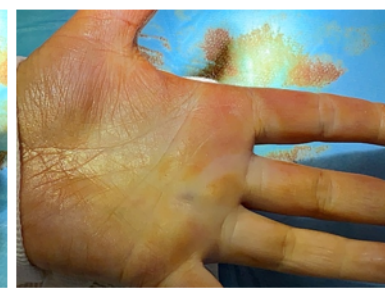

(d)
Figure 1: (a) Trigger ring digit right hand (black arrow), (b) Lance knife 19 Gauge (black arrow), (c) Full flexion of the ring digit, (d) Full extension of the digit.

$<0,05$ considered to be statistically significant) and Fishers exact test was performed to assess the outcomes ( $p<0,05$ considered to be statistically significant).

Results

The two groups were similar regarding sex, age, type of trigger finger (solitary or multiple), duration of symptoms prior release, diabetes mellitus (Type I or II), hand side (dominant or non-dominant) and grade of triggering (Table 2). Mean follow-up is 12 months (range from 10 months to 14 months). Patients were re-examinated in two weeks, four weeks, three and six months and finally at one year. The short-term results referring to triggering, pain, stiffness, scar, correspond to three months duration and satisfaction ( $p$ value $=0,010$ ) while the long-term results to one year ( $p$ value $=0,9$ ) (Table 3 and 4 ). Before releasing patients from both groups, VAS score was taken, while after operation and in reassessment visits, the questionnaire form of VAS, Quick Dash and Gilbert and Wereldasma were fulfilled for. At the last follow-up, in one year postoperatively, we used a phone call communication. Among complications, in group A five cases had an infection (three cases of them with multiples digits) in which we encountered antibiotic therapy, eight cases had pain and scar formation and three cases had a metacarpophalangeal joint stiffness. For resolving the last two complications, patients received a physiotherapy program and painkillers. In P.R. group we had four cases with incomplete release in four patients (two indexes, one thumb and one ring digit). These patients had undergone an open release of A1 pulley after two weeks. We had three cases with transient radial nerve injuries in thumb, which were resolved at three and four weeks. Finally, in three cases we had a slight hematoma (two in index finger and one in ring digit). In short-term period the results in Group B (VAS score 0,58, Quick Dash Score 6,8 and Satisfaction Rate 67,9\%) seemed to be better than in Group A (VAS score 1,32, Quick Dash Score 9,1 and Satisfaction Rate 57,7\%) (Table 3). In long-term period (three, six months) the results in open release group seemed

\begin{tabular}{|l|c|c|c|}
\hline \multicolumn{1}{|c|}{ Characteristics } & Group A & Group B & P \\
\hline Triggering & 6 & 3 & 0,8 \\
Pain & 3 & 2 & 0,73 \\
Stiffness & 3 & 0 & 0,04 \\
Digital Nerve Injuries & 0 & 3 & 0,19 \\
Scar & 8 & 0 & 0,045 \\
Satisfaction & & & \\
Dissatisfied & $10(19,2 \%)$ & $6(9,6 \%)$ & \\
Satisfied & $12(23,1 \%)$ & $14(22,5 \%)$ & 0,010 \\
Very Satisfied & $30(57,7 \%)$ & $42(67,9 \%)$ & \\
\hline
\end{tabular}

Table 3: Short-Term results (3 months) Gilberts and Wereldasma questionnaire.

Group a. open release; Group b. percutaneous release.

\begin{tabular}{|l|c|c|c|}
\hline \multicolumn{1}{|c|}{ Characteristics } & Group A & Group B & P \\
\hline Triggering & 0 & 0 & N.C. \\
Pain & 1 & 1 & 0,1 \\
Stiffness & 1 & 0 & 0.277 \\
Digital Nerve Injuries & 0 & 0 & N.C. \\
Scar & 2 & 0 & 0,217 \\
Satisfaction & & & \\
Dissatisfied & $2(3,8 \%)$ & $1(1,7 \%)$ & \\
Satisfied & $6(11,6 \%)$ & $8(12,9 \%)$ & 0,918 \\
Very Satisfied & $44(84,6 \%)$ & $53(85,4 \%)$ & \\
\hline
\end{tabular}

Table 4: Long-Term results (1 year) Gilberts and Wereldasma questionnaire.

Group A. Open Release Group; B. Percutaneous Release; N.C.: Not Computable.

to be better and finally in one year both groups did not have any significant difference (Table 4).

\section{Discussion}

Trigger finger or Stenosing Tenosynovitis appears with painful catching of the flexor tendons during motion of flexion-extension of the affected digit. Whereas the disorder is progressive the digit locks in flexion with restricted range of motion. Finally, over time and because of patient's pain the disorder leads to fixed deformity at the proximal interphalangeal joint (PIP). Diabetic patients display a greater predisposition to develop trigger finger compared with non-diabetic population, while their symptoms are presented more severe, more than one digit are affected, and have bilateral involvement [12,14]. Abate M., et al. supported that progression of diabetic trigger finger comes from the coexistence of cheiroarthropathy which further limits hand mobility [13]. Duration of diabetes, age of the patient, and glucose control are thought to affect the severity of symptoms, therefore, strict glucose control is a necessary component of treatment [23].

In literature, several surgical or nonsurgical therapeutic protocols have been reported. We agree with the opinion that the deci- 
sion on how treating a patient with a trigger digit is often based on personal preference rather than on scientific verifications [17]. Nonsurgical options include NSAIDs, splinting and steroid injection with a successful rate in $57 \%$ to $97 \%$ of the cases [15]. Colbourn J., et al. reported relief of symptoms by splinting metacarpophalangeal joint with custom made splint for 6 - 10 weeks. In literature the corticosteroid injection has been reported to be effective after one injection in about half of cases and after the second injection the rate is up to $86 \%$ [18]. Successful results of steroid injection, related to patients who had been symptomatic less than a month, were higher than in cases who had been symptomatic for 6 months or more (88\% versus 65\%) [19]. Complication rates of the method have been reported to be up to $29 \%$ when symptoms persist more than six months [20]. Diabetes Mellitus is a bad prognostic factor which affects adversely the results of the method and leads to operative technique if failure occurs within one year [21].

Operative procedure has a purpose on surgical release of A1 pulley by open (oblique incision or straight incision) or percutaneous approach, nevertheless split of the affected pulley has complications. Open release of A1 pulley remains the mainstay of the treatment for trigger finger with satisfactory results of $60 \%$ $100 \%$. Whatever incidence of complications exists, related to open release (infections, pain, scar formation, joint stiffness or weakness, bowstringing of the flexor tendon, digital nerve or artery injuries) rates from $7 \%$ to $29 \%[19,22]$. Brown., et al. reported that in diabetic trigger finger, open surgical release is less effective and causes more pain in diabetic patients compared with non-diabetics, while Stahl., et al. reported that in 120 patients there was a success rate of $77 \%$ in diabetics and $94 \%$ in non-diabetics [23]

Lorthioir J (1958) firstly described the percutaneous release (P.R.) of trigger finger using a fine tenotome in 52 patients without neurovascular complication, and supported that the technique is effective, convenient, safe and well tolerated by patients [18]. Until today various modifications of the surgical instruments (fine scalpel, curved knife blade, HAKI knife, handle knife) have been described and the majority of them presented good functional results with an incidence of 94,4\% [25]. Eastwood., et al. reported a 94\% success rate in 35 trigger fingers with percutaneous release of A1 pulley, using a 21 gauge needle and suggested that the main advantage of percutaneous release is that is less invasive technique and minimizes all the complications of open release. The incidence of successful functional outcomes with gauge needle varies from $84 \%$ - $97 \%$ in recently reported literature.

Bain., et al. suggested that the method is not recommended for the thumb and index finger, because of the proximity of radial digital nerve, and for patients with locking trigger fingers, while Uras., et al. advised caution in thumb access, for the danger of the digital nerve injury [26]. Guler., et al. reported digital nerve injuries of trigger thumb with an incidence of 5,7\%. Maneerit., et al. suggested that in order to avoid the digital nerve injury of the thumb: I) the needle must be in the midline position of the flexor tendon,
II) the thumb must be in full extension, III) the forearm should be placed in hypersupination, to place the palmar surface of thumb in a horizontal plane for good orientation and IV) the needle must be inserted a few millimeters distal to the metacarpophalangeal flexion crease. In our series we had 3 cases (thumb) with transient radial nerve injuries which were resolved in a mean period of two months.

Other complications of the P.R. method reported include persistent pain, recurrent triggering, restriction of metacarpophalangeal motion, tendon rupture, hypoesthesia, bowstringing and hematoma [26]. The main reason of postoperative pain is the incomplete release of pulley and tendon laceration or rupture. Incidence of incomplete release of A1 pulley varied between 10\% - 15\%. Gilberts. et al. mentioned that incomplete release could existed even though the triggering had disappeared postoperatively, while Calleja., et al. supported that complete anatomic release of the A1 pulley is not always adequately achieved, even though patients experience relief of triggering clinically [28]. Wojahn., et al. referred that painful tenosynovitis can occur from laceration of the flexor tendon after P.R. and in these cases corticosteroid injection can relieve the symptoms [26]. Saremi., et al. suggested that percutaneous release with corticosteroid injection in diabetic trigger finger seemed to have better functional outcomes, while Kuczmarski., et al. mentioned that P.R. with or without simultaneous corticosteroid injection in the treatment of diabetic trigger finger showed promising results [18,23]. In our series we had 3 cases with persistent pain at 4 weeks postoperatively, which required open release for incomplete release of A1 pulley ( 2 thumbs and one middle finger). We believe that the cause is the insufficient experience of the method in initial cases. As painful tenosynovitis concerned, there was no case in our series and we believe that corticosteroid injection played a role in healing process of the flexor tendon without being able to substantiate it.

Gilberts., et al. compared open release versus percutaneous in 100 trigger digits according to operation time, postoperative pain and recovery time of motor function and reported successful results of $98 \%$ in group of open release and $100 \%$ in cases with percutaneous technique. Operation time was significant longer in open release while pain and time to functional recovery were shorter in percutaneous release with no serious complications in either group [27]. Adhav., et al. reported that advantages of percutaneous release are, less postoperative morbidity, less risk of recurrence, no blood loss, no fibrosis [27]. Publications of cost analysis have shown that treating diabetic trigger finger with percutaneous technique is more cost-effective method than open release $[17,23]$

Lin., et al. compared the degree of satisfaction in short and long-term outcomes between open and P.R. and mentioned that the functional outcomes and satisfaction in short-term are significantly better in P.R. group, while in long-term, there were no differences between the two groups [28]. The same conclusion came out in our series in which pain (VAS score), functional scores (Quick dash score, Gilbert and Wereldasma questionnaire) and complica- 
tion rates are better for P.R. group in three months than the open release group, while in one year the results appeared with no statistical differences.

However, our study has two limitations. First the duration of the follow-up period should be bigger and second the sample is not enough to ensure sufficient statistically improvement.

\section{Conclusion}

Trigger finger is a disorder which implicates the function of the hand in daily living activities and appears with more severity of symptoms in diabetic patients. When diabetic trigger finger is unresponsive to conservative treatment, the release of A1 pulley is mandatory. Open and percutaneous release are two methods in which long-term outcomes do not differ significantly, but in shortterm, the percutaneous release is reliable, cost effective technique, if performed carefully, with good recovery of function and patient satisfaction.

\section{Conflict of Interest}

The authors declare that have no conflict of interest.

\section{Bibliography}

1. Yadav A., et al. "Comparison between Percutaneous release and Corticosteroid injection in the management of trigger digits". International Journal of Orthopaedics Sciences 3.3 (2017): 21-26.

2. Saldana M. "Trigger digits: diagnosis and treatment". The Journal of the American Academy of Orthopaedic Surgeons 9 (2001): 246-252.

3. Fitzgerald B., et al. "Gout affecting the hand and wrist". The Journal of the American Academy of Orthopaedic Surgeons 15 (2007): 625-635.

4. Freiberg A., et al. "Nonoperative treatment of trigger fingers and thumbs". The Journal of Hand Surgery American 14.3 (1989): 553-558.

5. Langer D., et al. "Evaluating hand function in clients with trigger finger". Occupational Therapy International (2017): 9539206.

6. Brozovich N., et al. "A Critical Appraisal of Adult Trigger Finger: Pathophysiology, Treatment, and Future Outlook". Plastic and Reconstructive Surgery 7 (2019): e2360.

7. Wiwanitkit $\mathrm{S}$ and Wiwanitkit V. "Trigger Digits and Diabetes Mellitus". North American Journal of Medical Sciences 4.3 (2012): 117-119.

8. Hansen RL., et al. "Open surgery versus ultrasound-guided corticosteroid injection for trigger finger: a randomized controlled trial with 1-year follow-up". The Journal of Hand Surgery American 42 (2017): 359-366.
9. Hoang D., et al. "Evaluation of percutaneous first annular pulley release: efficacy and complications in a perfused cadaveric study". The Journal of Hand Surgery 41 (2016): e165-e173.

10. Wilhelmi BJ., et al. "Trigger finger release with hand surface landmark ratios: an anatomic and clinical study". Plastic and Reconstructive Surgery 108.4 (2001): 908-915.

11. Hazani R., et al. "Assessment of the distal extent of the A1 pulley release: a new technique". Eplasty 22.8 (2008): e44.

12. Vance MC., et al. "The association of hemoglobin A1c with the prevalence of stenosing flexor tenosynovitis". The Journal of Hand Surgery 37.9 (2012): 1765-1769.

13. Abate M., et al. "Management of limited joint mobility in diabetic patients". Diabetes, Metabolic Syndrome and Obesity Targets 6 (2013): 197-207.

14. Ferree S., et al. "Risk factors for return with a second trigger digit". The Journal of Hand Surgery European 39 (2014): 704707.

15. Nimigan AS., et al. "Steroid injections in the management of trigger fingers". American Journal of Physical Medicine and Rehabilitation 85 (2006): 36-43.

16. Colbourn J., et al. "Effectiveness of splinting for the treatment of trigger finger". Journal of Hand Therapy 21.4 (2008): 336343.

17. Monreal R., et al. "Percutaneous Trigger Finger Release in Outpatient Clinics". Clinical Research in Orthopaedic 1.2 (2018): $1-3$.

18. Saremi H., et al. "Percutaneous Release of Trigger Fingers: Comparing Multiple Digits with Single Digit Involvement". The Archives of Bone and Joint Surgery 4.3 (2016): 224-227.

19. Turowski GA., et al. "The results of surgical treatment of trigger finger". The Journal of Hand Surgery 22A (1997): 145-149.

20. Sato ES., et al. "Percutaneous release of trigger fingers". Hand Clinics 30.1 (2014): 39-45.

21. Sheikh E., et al. "A prospective randomized trial comparing the effectiveness of one versus two (staged) corticosteroid injections for the treatment of stenosing tenosynovitis". Hand (NY) 9 (2014): 340-345.

22. Sahu RL and Gupta P. "Experience of percutaneous trigger finger release under local anesthesia in the medical college of Mullana, Ambala, Haryana". Annals of Medical and Health Science Research 4.5 (2015): 806.

23. Kuczmarski AS., et al. "Management of diabetic trigger finger". Journal of Hand Surgery American 44.2 (2019): 150-153. 
24. Brown E and Genoway KA. "Impact of diabetes on outcomes in hand surgery". The Journal of Hand Surgery American 36.12 (2011): 2067-2072.

25. Pandey BK., et al. "Percutaneous trigger finger release". Nepal Orthopaedic Association Journal 1.1 (2010): 25-29.

26. Aksoy A and Sir E. "Complications of Percutaneous Release of the Trigger Finger". Cureus 11.2 (2019): e4132.

27. Elsayed MM. "Percutaneous release of trigger finger". The Egyptian Orthopaedic Association 48.3 (2013): 277-281.

28. Lin AJ., et al. "Open versus percutaneous release for trigger digits: Reversal between short - term and long-term outcomes". Journal of the Chinese Medical Association 79 (2016): 340-344.

\section{Assets from publication with us}

- Prompt Acknowledgement after receiving the article

- Thorough Double blinded peer review

- Rapid Publication

- Issue of Publication Certificate

- High visibility of your Published work

Website: https://www.actascientific.com/

Submit Article: https://www.actascientific.com/submission.php Email us: editor@actascientific.com

Contact us: +919182824667 